\title{
Hacia una mayor incorporación de la perspectiva del paciente en el diseño de los instrumentos de evaluación de la efectividad y calidad de los cuidados
}

\author{
M. Romero-García ${ }^{a, b}$ y J. Trujols-Albet ${ }^{c}$ \\ a Departamento de Enfermería Fundamental y Medicoquirúrgica, Escuela \\ Universitaria de Enfermería. Universidad de Barcelona, Barcelona, España. \\ ${ }^{b}$ GRIN-IDIBELL (Grupo de Investigación Enfermera del Instituto de \\ Investigación Biomédica de Bellvitge). \\ c Servicio de Psiquiatría, Hospital de la Santa Creu i Sant Pau, Barcelona, \\ España.
}

A lo largo de las últimas décadas, se ha producido un progresivo cambio de paradigma donde el paciente deja de ser un mero objeto de la atención sanitaria para convertirse en un sujeto activo de la misma y paralelamente se empieza a dar más importancia a los cuidados centrados en el paciente. El abandono de actitudes paternalistas favorece que el objetivo principal de la prestación de un cuidado sea el paciente, haciéndose imprescindible conocer su opinión para poder evidenciar indicadores de contexto, procesos y resultados que de otra manera serían difícilmente accesibles. Dicho conocimiento posibilita a su vez poder ajustar el servicio ofertado a las necesidades, expectativas y prioridades del paciente. En este sentido, la actualización continua de conocimientos y el desarrollo de actitudes y habilidades encaminadas a la satisfacción de las necesidades del paciente y sus expectativas se convierte en un gran reto tanto para los profesionales como para las organizaciones sanitarias.

Cada vez más, las enfermeras somos conocedoras de la importancia de escuchar a los pacientes, y esto se ve reflejado en la proliferación de estudios mixtos, donde se combina la metodología cualitativa con la cuantitativa. Accediendo y visibilizando la perspectiva de los pacientes, las enfermeras podemos mejorar la calidad de los cuidados, ya que las percepciones de los pacientes aportan nuevos datos a una misma realidad ${ }^{(1)}$. Nuestra práctica debe 
asentarse en la mejor evidencia disponible tanto sobre la efectividad de nuestras intervenciones como acerca de las necesidades, prioridades y experiencias de los pacientes. De hecho, las guías de práctica clínica enfatizan cada vez más la consideración y respeto de los valores y prioridades de los pacientes.

Para medir la calidad o la satisfacción con los cuidados enfermeros disponemos de instrumentos de medida pero ¿Quién los ha diseñado? ¿Desde qué perspectiva? ¿No sería más lógico incorporar la perspectiva del paciente también en el diseño de dichos instrumentos en lugar de auto-otorgarnos el rol de decidir qué dimensiones e indicadores configuran dicho constructo de satisfacción o calidad desde el punto de vista del paciente?

En esta línea, recientemente se ha propuesto una clasificación de los instrumentos de medida en función de su nivel de reconocimiento e incorporación de la perspectiva del paciente. Esta taxonomía, formulada originalmente para los instrumentos de medida utilizados en la evaluación de resultados ${ }^{(2)}$, clasifica los cuestionarios de evaluación de la satisfacción con las intervenciones enfermeras en 4 categorías $\operatorname{distintas}^{(3)}$ :

1. Cuestionario de Satisfacción del Paciente-Generado por los Pacientes (CSP$G P)$. Se trata de un instrumento elaborado y desarrollado íntegramente desde la perspectiva de los pacientes. Su principal característica diferencial radica en el hecho de que todas las personas implicadas en las diferentes fases de su construcción y desarrollo son o han sido receptores de la intervención o los cuidados acerca de los que se va a evaluar su satisfacción.

2. Cuestionario de Satisfacción del Paciente-Centrado en el Paciente (CSP-CP). Consiste en un CSP desarrollado incorporando, explícitamente y en mayor o menor grado, la perspectiva de los pacientes utilizando, como en el caso de los CSP-GP, una metodología mixta. En un primer momento, los grupos focales o las entrevistas en profundidad son imprescindibles para, desde la perspectiva de los pacientes, generar las dimensiones y variables a evaluar o redactar los correspondientes ítems. En una segunda fase, se siguen las habituales aproximaciones cuantitativas encaminadas a delimitar las propiedades psicométricas del instrumento. Un ejemplo pertinente de este tipo de 
cuestionarios de satisfacción es una escala de satisfacción con los cuidados enfermeros en pacientes críticos, actualmente en su fase final de desarrollo en nuestro ámbito geográfico de referencia ${ }^{(4,5)}$.

3. Cuestionario de Satisfacción del Paciente-Valorado por el Paciente (CSP-VP). En esta tercera categoría se incluyen aquellos CSP cuyo contenido, a pesar de haber sido elaborado sin la participación de los pacientes, es valorado positivamente por una mayoría de ellos al reflejar, al menos en cierta medida, sus propias perspectivas ${ }^{(6)}$. En otras palabras, los pacientes consideran que los CSP-VP han sido desarrollados por alguien que posee un buen conocimiento de la realidad de los propios pacientes.

4. Cuestionario de Satisfacción del Paciente-Irrelevante para el Paciente (CSPIP). Consiste en un CSP desarrollado sin ningún tipo de participación directa de los pacientes y evaluado como no relevante para $-\mathrm{y}$ por parte de- ellos al no medir factores que ellos consideran importantes.

Siguiendo esta clasificación, en el desarrollo de todo Cuestionario de Satisfacción del Paciente-Generado por los Pacientes (CSP-GP), los propios pacientes se convierten en investigadores y lideran la investigación, necesitándose para ello pacientes formados en metodologías y técnicas de investigación. Un protocolo estandarizado para el desarrollo de este tipo de instrumentos de medida en el ámbito de la salud mental ha sido publicado recientemente $^{(7)}$.

Por otro lado, cabe señalar que los resultados obtenidos mediante la utilización de cualquier Cuestionario de Satisfacción del Paciente-Irrelevante para el Paciente (CSP-IP), son cuestionables al no incorporar dichos instrumentos aquellas cuestiones y temáticas importantes para los pacientes. En este sentido algunos autores destacan que "la búsqueda de la virtud psicométrica es inútil si el instrumento está tan mal enfocado que es irrelevante para el paciente"(8).

Sin duda alguna, nadie está mejor situado que el paciente mismo a la hora de aprehender su propio punto de vista sobre la experiencia y el resultado de unos cuidados o unas intervenciones. Por ello solo mirando a través de los ojos del paciente veremos cómo mejorar los cuidados que dispensamos. Posibilitar unos 
cuidados realmente centrados en el paciente requiere una evaluación de dichos cuidados igualmente centrada en el paciente. Dicha evaluación únicamente será posible si, entre otros requisitos como el de la robustez psicométrica, se considera el grado de incorporación de la perspectiva de los pacientes por parte del instrumento antes de seleccionar alguno entre los disponibles, o se estima previamente el grado de sensibilidad hacia dicha perspectiva en el caso de que únicamente existan cuestionarios desarrollados sin la participación directa de los pacientes. Asimismo, si el objetivo de alguna de vuestras investigaciones futuras fuera desarrollar un nuevo instrumento de medida, dicho grado de incorporación de la perspectiva del paciente es un aspecto primordial a considerar y fortalecer.

\section{Bibliografía}

1. Regaira Martínez E, Sola Iriarte M, Goñi Viguria R, Del Barrio Linares M, Margall Coscojuela MA, Asiain Erro MC. La calidad asistencial en cuidados intensivos evaluada por los pacientes mediante la escala SERVQUAL. Enferm Intensiva. 2010;21(1):3-10.

2. Trujols J, Portella MJ, Iraurgi I, Campins MJ, Siñol N, Pérez de los Cobos J. Patient-reported outcome measures: are they patient-generated, patient-centred or patient-valued? J Ment Health. 2013;22(6):555-62.

3. Trujols J, Iraurgi I, Oviedo-Joekes E, Guàrdia-Olmos J. A critical analysis of user satisfaction surveys in addiction services: opioid maintenance treatment as a representative case study. Patient Prefer Adherence. 2014;8:107-17.

4. Romero-García M, de la Cueva-Ariza L, Jover-Sancho C, Delgado-Hito P, Acosta-Mejuto $B$, Solà-Ribó $M$, et al. La percepción del paciente crítico sobre los cuidados enfermeros: una aproximación al concepto de satisfacción. Enferm Intensiva. 2013; 24(2):51-62.

5. de la Cueva-Ariza L, Romero-García M, Delgado-Hito P, Acosta-Mejuto B, Jover-Sancho C, Ricart-Basagaña MT, et al. Development of an 
instrument to measure the degree of critical patient's satisfaction with nursing care: research protocol. J Adv Nurs. 2014; 70(1):201-10.

6. Kabir T, Wikes, T. Measures of outcomes that are valued by service users. En: Thornicroft G, Tansella M, editores. Mental health outcome measures ( $3^{a}$ ed). London: Royal College of Psychiatrists; 2010. p. 3-14.

7. Rose D, Evans J, Sweeney A, Wykes TA. Model for developing outcome measures from the perspectives of mental health service users. Int Rev Psychiatry. 2011; 23(1): 41-6.

8. Bilsbury CD, Richman A. A staging approach to measuring patientcentred subjective outcomes. Acta Psychiat Scand. 2002;106 (Suppl. 414):5-40. 